\title{
Global quantification of intact proteins via chemical isotope labeling and mass
}

\section{spectrometry}

Zheyi Liu ${ }^{1, \#}$, Ruimin Wang ${ }^{2, \#}$, Jing Liu ${ }^{3}$, Ruixiang Sun ${ }^{2, *}$, Fangjun Wang1,*

1 CAS Key Laboratory of Separation Sciences for Analytical Chemistry, Dalian Institute of Chemical Physics, Chinese Academy of Sciences, Dalian, 116023, China

2 Institute of Computing Technology, Chinese Academy of Sciences, Beijing, 100190, China

3 College of Pharmacy, Dalian Medical University, Dalian, 116044, China

\# Contributed equally to this work.

* Corresponding Authors (Email: wangfj@dicp.ac.cn and rxsun@ict.ac.cn)

Dedicated to the 70th anniversary of Dalian Institute of Chemical Physics, CAS

\section{Contents:}

\section{- Supportting exprimental}

- Figure S1 The overlay spectra of unlabeled and labeled lysozyme (A) and RNase (B).

- Figure S2 The fragmentation patterns of myoglobin ions with different charge state.

- Figure S3 The fragmentation patterns of lysozyme ions with different charge state.

- $\quad$ Figure S4 The MS spectra of dimethyl isotope labeled proteins (heavy/light =1), (A) RNase A; (B) lysozyme; (C) myoglobin and (D) $\beta$-casein.

- $\quad$ Figure S5 The MS spectra of dimethyl labeled $\beta$-casein. (A) the ion pairs with different charges in 1:1 pre-mixed sample; (B-E) the MS spectra of 1:1, 2:1, 5:1 and 10:1 with the charge state of 17+.

- Figure S6 The measured and calculated MS spectra of myoglobin with a charge state of $17+$. The monoisotopic peak was indicated by red arrow.

- Figure S7 The linearity plot of quantitative ratio of each intact protein.

- $\quad$ Figure S8 The quantification ratios of the mixed intact proteins with different pre-mixing ratios based on the intensities of monoisotopic species after deconvolution.

- Figure S9 (A) MS1 spectra of an unknown protein with a mass of $28 \mathrm{kDa}$ detected in both A and B group; (B) the enlarge figures of MS1 spectra and a consistent result is obtained for this protein, which indicates that high quantification accuracy could well be obtained for large proteins.

- Figure S10 the MS1 and MS2 spectra of an unknown protein with a mass of $28 \mathrm{kDa}$.

- Figure S11 The correlation of quantification ratios $(\log 2)$ for the proteoforms in groups A and B.

- $\quad$ Figure S12 The quantification of GRE1 protein in Group A: (A), the MS1 spectra of GRE1 protein; (B), annotated and deconvoluted spectra of GRE1 protein.

- Figure S13 The quantification of HSP12 protein in Group A: (A), the MS1 spectra of HSP12 protein; (B), annotated and deconvoluted spectra of HSP12 protein.

- Table S1 The identified peptides from HeLa proteome after dimethyl labeling.

- Table S2 The up-regulated and down-regulated proteins (anaerobic vs aerobic).

- Table S3 The quantified proteoforms from yeast proteome under anaerobic and aerobic conditions. 


\section{Experimental}

The detailed GELFrEE program

\begin{tabular}{|c|c|c|c|c|c|}
\hline Step & Voltage & Time (min:sec) & Step & Voltage & Time (min:sec) \\
\hline 1 & $50 \mathrm{~V}$ & $16: 00$ & 8 & $100 \mathrm{~V}$ & $2: 48$ \\
\hline 2 & $50 \mathrm{~V}$ & $44: 00$ & 9 & $100 \mathrm{~V}$ & $3: 18$ \\
\hline 3 & $50 \mathrm{~V}$ & $3: 00$ & 10 & $100 \mathrm{~V}$ & $4: 00$ \\
\hline 4 & $50 \mathrm{~V}$ & $3: 36$ & 11 & $100 \mathrm{~V}$ & $7: 00$ \\
\hline 5 & $50 \mathrm{~V}$ & $4: 00$ & 12 & $100 \mathrm{~V}$ & $8: 00$ \\
\hline 6 & $100 \mathrm{~V}$ & $2: 18$ & 13 & $100 \mathrm{~V}$ & $20: 00$ \\
\hline 7 & $100 \mathrm{~V}$ & $2: 30$ & & & \\
\hline
\end{tabular}

\section{Investigation of labeling efficiency by bottom-up proteomics analysis}

Preparation of the HeLa proteins. The HeLa cell was cultured in RPMI medium with $10 \%$ newborn bovine serum (BS) and penicillin/streptomycin $(1 \times)$ at $37{ }^{\circ} \mathrm{C}$ in $5 \% \mathrm{CO}_{2}$. Until the cell grown to about $90 \%$ of the petri dish, the RPMI was removed and the cells were washed with cold PBS twice. Then the cells were treated with trypsin and collected in a $10 \mathrm{~mL}$ centrifuge tube. The cells pellets were softly homogenized in an ice-cold lysis buffer containing $8 \mathrm{M}$ urea, $50 \mathrm{mM}$ Tris- $\mathrm{HCl}(\mathrm{pH}$ 7.4), $65 \mathrm{mM}$ DTT, 2\% protease cocktail (v/v), 1\% Triton X-100 (v/v), 1 mM EDTA, 1 mM EDGA and 1 $\mathrm{mM}$ PMSF assisted by an intense sonication $(400 \mathrm{~W} \times 60 \mathrm{~s} \times 3)$. The cell lysate was centrifuged for $30 \mathrm{~min}$ at $25000 \mathrm{~g}$ and $4{ }^{\circ} \mathrm{C}$, and supernatant was treated with 5 times volume of ice-cold protein precipitation solution and kept at $-30{ }^{\circ} \mathrm{C}$ overnight. The precipitated protein was obtained by centrifugation for $30 \mathrm{~min}$ at $25000 \mathrm{~g}$ and $4{ }^{\circ} \mathrm{C}$, washed stepwise with $5 \mathrm{~mL}$ ice-cold acetone and $75 \%$ ethanol. The HeLa proteins was dissolved in $6 \mathrm{M}$ Urea and $50 \mathrm{mM}$ Hepes (pH 7.4) and dimethylated by pyridine borane and formaldehyde as mentioned in the manuscript. The dimethylated HeLa lysate was reduced and alkylated by DTT and IAA, then digested by trypsin at $37^{\circ} \mathrm{C}$ overnight. The HeLa digest was desalted by SEP column, lyophilized and stored at $-20^{\circ} \mathrm{C}$ before use.

LC-MS/MS analysis of HeLa peptides. The bottom up analysis of Hela peptides was analyzed by Q Exactive (Thermo Scientific, Waltham, MA). The HeLa peptides was re-dissolved in 0.1\% FA (Buffer A). 1 ug Hela peptides were loaded to a $3 \mathrm{~cm} \times 200 \mu \mathrm{m}$ trap column packed with C18 AQ beads (Sunchrom, $5 \mu \mathrm{m}, 120 \AA$ ) with Buffer A at $5 \mu \mathrm{L} / \mathrm{min}$, and separated by a $15 \mathrm{~cm} \times 75 \mu \mathrm{m}$ C18 column (same beads with trap column) at $300 \mathrm{~nL} / \mathrm{min}$ with a gradient of $5 \sim 40 \%$ Buffer B $(80 \% \mathrm{ACN}, 0.1 \% \mathrm{FA})$ in $60 \mathrm{~min}$. The full scan was obtained at a resolution of $70000(\mathrm{~m} / \mathrm{z}=200)$ with an AGC of $1 \times 10^{6}$, and the Top 15 ions (intensity above 1e4) was subjected to HCD fragmentation at a normalized collision energy of 28 with a constant injection time of $60 \mathrm{~ms}$. The product ion was analysis with a resolution of $17500(\mathrm{~m} / \mathrm{z}=200)$. The dynamic exclusion was enabled with a value of $30 \mathrm{~s}$ and the peptides ions with charge state (unassigned, 1 or above 8) were also excluded for MS/MS.

Date analysis. The Data of dimethylated peptides were searched against human database (uniprot, version 201307) by Thermo Proteome Discoverer (Thermo Scientific, Waltham, MA; version 1.4.1.14). Precursor and fragment mass tolerance were set at $10 \mathrm{ppm}$ and $0.05 \mathrm{Da}$, respectively. The Trypsin digestion was selected, and the max missed cleavage sites was set at 5 considering the miss 
cleavage caused by dimethylation. The static modification was carbamidomethyl cysteine and oxidation of methionine and dimethyl lysine was selected as dynamic modifications. Both the protein and peptides identification false positive rates were less than $1 \%$.

\section{Figures}
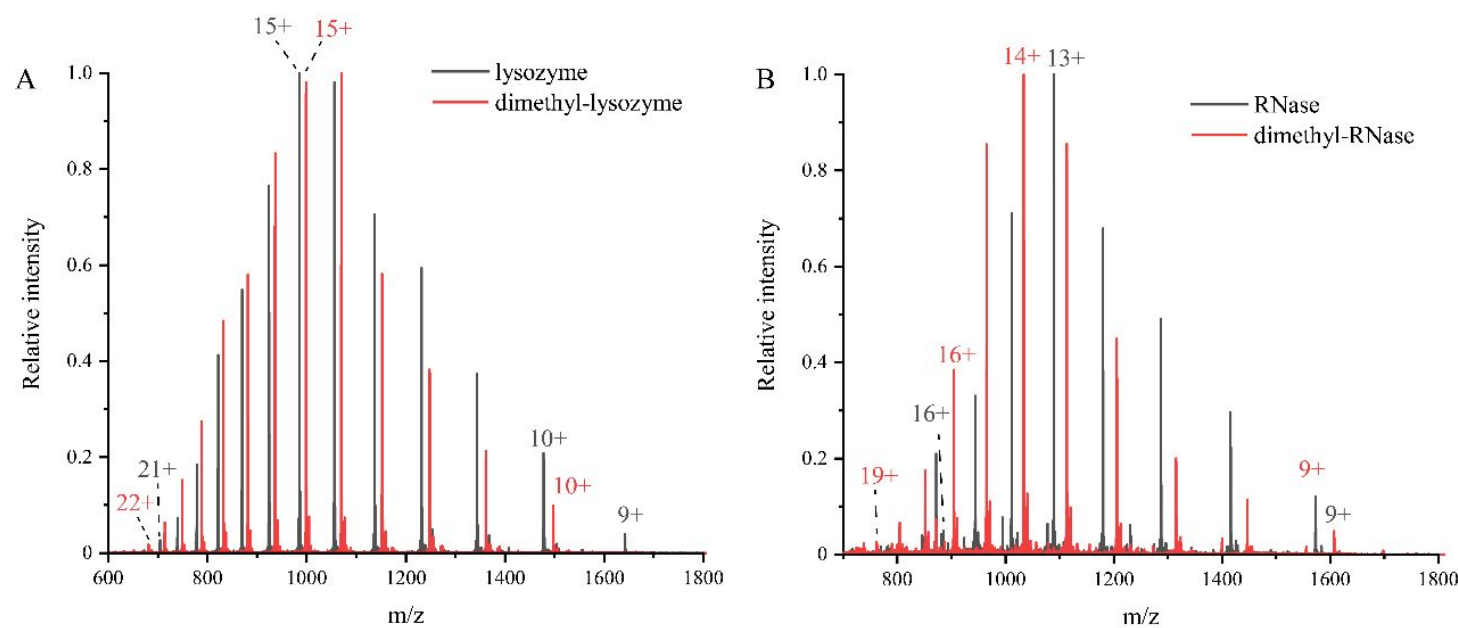

Figure S1 the overlay spectra of unlabeled and labeled lysozyme (A) and RNase (B). A charge enhancement of dimethylation is confirmed after comparing the charge distribution of proteins with/without dimethylation. As shown in follow figure, the charge distributions of both proteins shift to the high charge state. For dimethyl labeled lysozyme, the charge distribution range is $10+$ to $22+$, while the unlabeled lysozyme has a charge distribution range of $9+$ to $21+$. The average charge state for labeled and unlabeled lysozyme is 14.75 and 14.2, respectively. For RNase, the highest observed charge state shift from $16+$ to $19+$ after dimethylation. The dimethyl labeled RNase also has a higher average charge state with 13.82 over 12.41 . The above results indicate that the dimethyl labeled protein has a higher affinity to hydrogen ions, which is also consisted with the difference between the proton affinities of primary $\left(218.4 \mathrm{~kJ} / \mathrm{mol}\right.$ for $\left.\mathrm{CH}_{3} \mathrm{NH}_{2}\right)$ and tertiary amines $\left(228.6 \mathrm{~kJ} / \mathrm{mol}\right.$ for $\left.\left(\mathrm{CH}_{3}\right)_{3} \mathrm{~N}\right)$ ( G. Raabe et al, Calculation of the Proton Affinities of Primary, Secondary, and Tertiary Amines Using Semiempirical and ab initio Methods, Zeitschrift für Naturforschung A, 2000, pp. 687.). The dimethylation transforms the primary amine of lysine to the tertiary amine. 
a

Unlabeled myoglobin $13+$ Residual cleavage: $49 \%$

G L L S D D G E E W Q Q Q Q V V L L W N V W W G K V V E E A D D I I A A G G H G

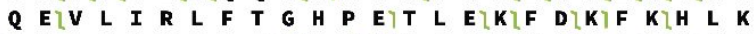

T E A E M K A S E D L K K K G T V V L L T A L G G G $L \backslash K \backslash K \backslash K \backslash G \backslash H\rceil H \quad E \backslash A \quad E \backslash L \backslash K \backslash P$ L A

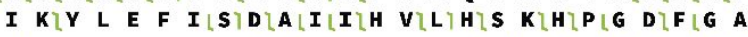

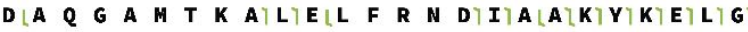

F) Q G C

Labeled myoglobin 13+ Residual cleavage: $38 \%$

G L S S D Q E E V L I R L F T G H P E T L E K F D K F K H L K T E A E E M K K A S S E E D L K K KLH G T V V L L T A $L K K\lfloor K\lfloor G$ H H E $\backslash A$ E

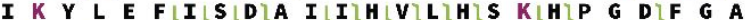
D A Q PIG A M T K K A L L E L F F R N D I A A K Y K K E L G F Q G C

b

Unlabcled myoglobin 15+ Residual clcavage: $32 \%$

G L S D D G E W W Q Q V L $L$ N V V W G K V E A D I A G H Q E E V L I R R L F T G H P T E A E E M K KLA S S E

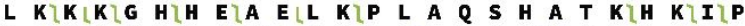

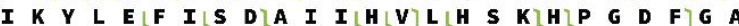

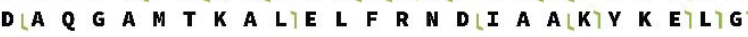
F Q G C

Labcled myoglobin $15+\quad$ Residual cleavage: $38 \%$

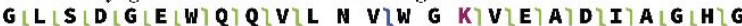
Q ELV L I R L F T G H PlE T L E K F D K K F K H L K T E)A E\M K A S E D L K KLH G T V V L T A L G G I L K K K K G G H H E A E K K P L A O S H A T K H K T I K K Y L L D $\backslash A$ Q $G$ G A M T K K A L E F $Q$ G C
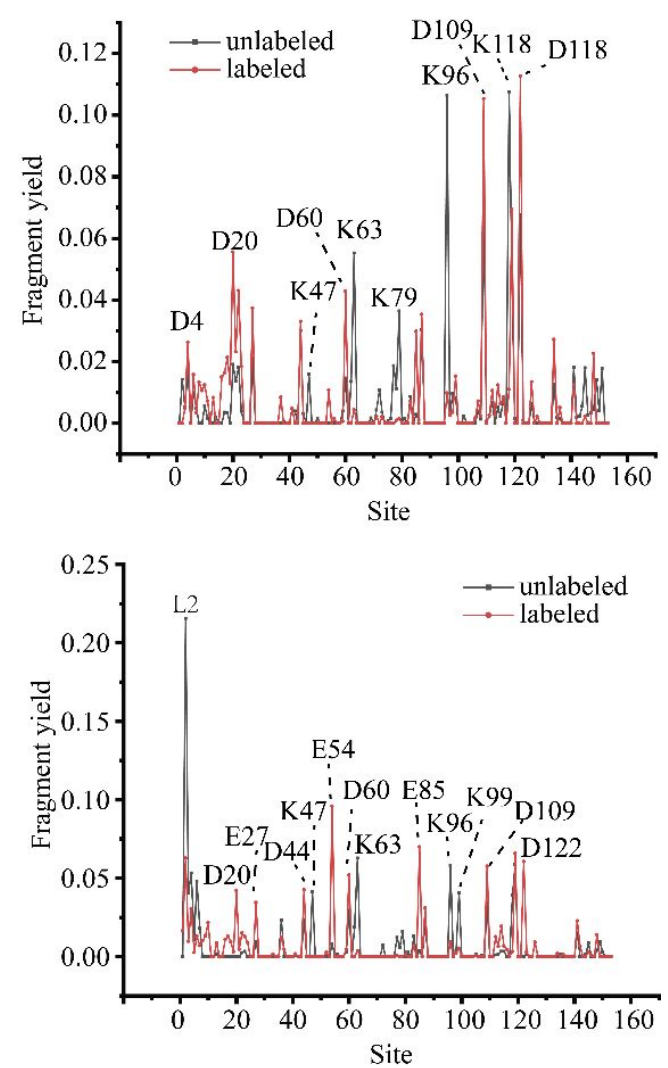

Figure S2 the fragmentation patterns of myoglobin ions with different charge state. The dimethylated and unlabeled myoglobin share a similar dissociation pattern at identical dissociation condition. No significant changes in dissociation patterns are observed for both protein ions with charge states $13+$ and $15+$ after dimethylation. However, a clear enchantment in dissociation tendency at acidic amino acids (D and E) is observed, while the dissociation at lysine is suppressed after dimethylation. 
a

Unlabeled lysozyme 14+ Residual cleavage: $41 \%$

KLV V F G R C E L L A A A A M K K R H G L D D N Y R G Y S L

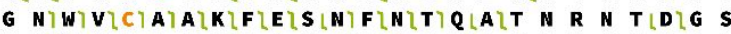

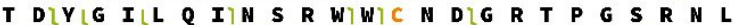

C $\mathbf{N}$ I $\backslash \mathbf{P}$ C C S S A A L L L L S S S D D I I T T A D G N G N A W V A WR NR C K G T D V OA W I R G C R L C

Labeled lysozyme 14+ Residual cleavage: $39 \%$

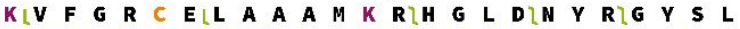
G N]W V V C A A

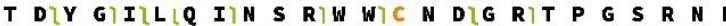

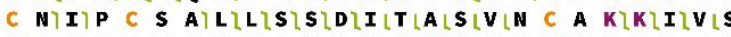
D $\backslash$ G N G M N A W V A W R N R TCLK G T D U V Q A W I R G C R L

$\mathbf{b}$

Unlabeled lysozyme 16+ Residual cleavage: $32 \%$

K V F G R C E L A A A M K R H G L D N Y R G Y S L

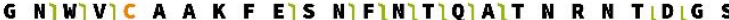

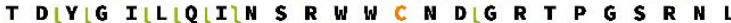

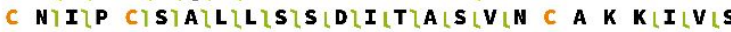
DLG N G MLN A W V A W R N R C K G T D V Q A W I R G C R L C

Labeled lysoryme $16+\quad$ Residual cleavage: $33 \%$

K V F F G R C E L A A A M K K R H G L D N Y

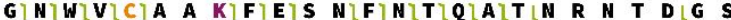
T:D Y Y G I L L I I I N S R W W C N D G R T P G S R

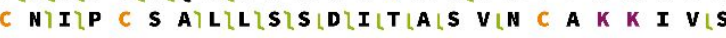
D G N G MLN A W V A W R N R C K G T D V Q A W I R

G C R L C

Unlabeled lysozyme $18+\quad$ Residual cleavage: $26 \%$

K V F G R C E L A A A M K R H G L D N Y R G Y S L

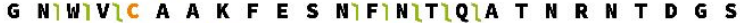

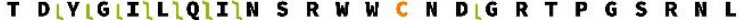

C N I I P C S $\mathbf{S}$ A L L L L S S S D I I T T A A S S V N

D G N G H N A W V A W R N R C K G T D V Q A A W I R

G C R L

Labeled lysozyme 18+ Residual cleavage: $27 \%$

K V F G R C E L A A A M K R H G L D N Y R G Y S L

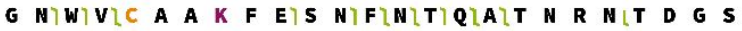

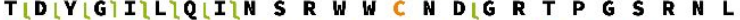
C N I P CIS A L LIS S D I T A S V N C A K K I V S DLG N G MLN A W V A W R N R C K G T DLV Q A W I R G $C \mathbf{R} \mathbf{L}$
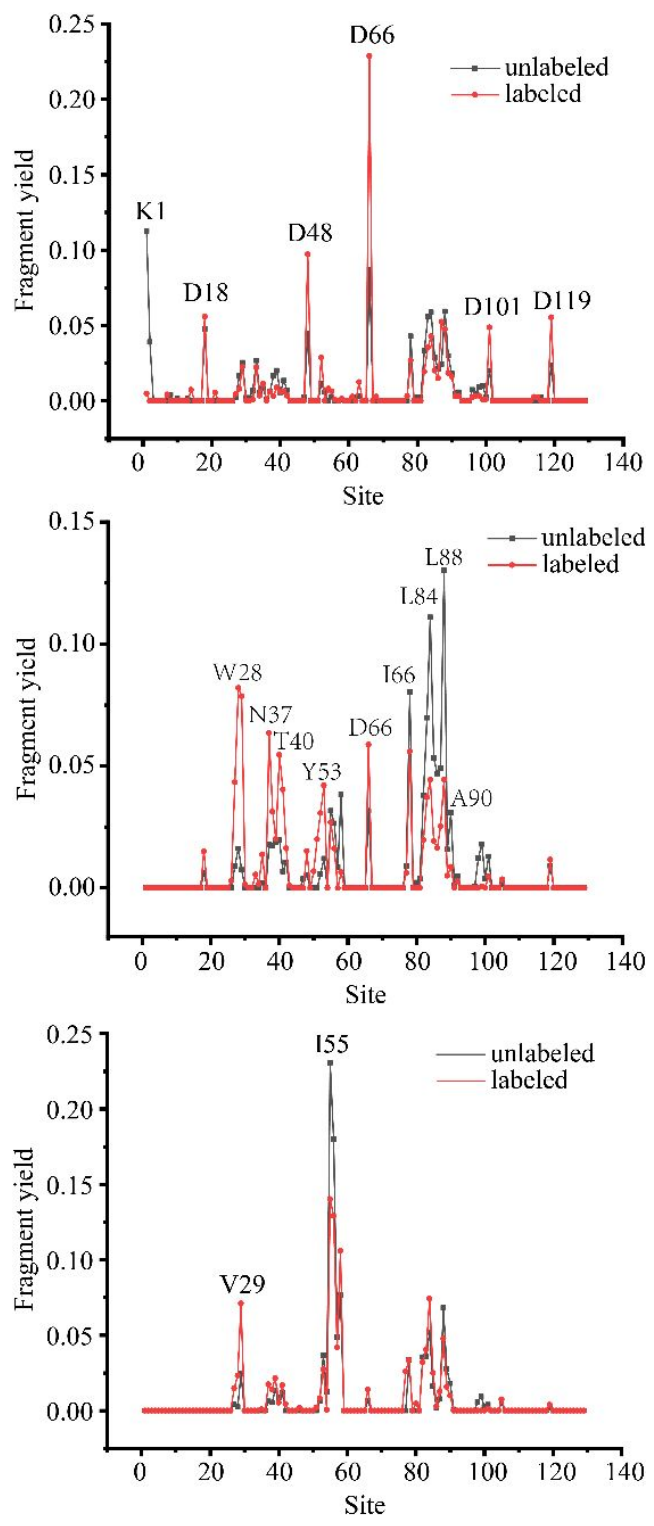

Figure $\mathrm{S} 3$ the fragmentation patterns of lysozyme ions with different charge state. The dissociation at aspartic acid residues (D18, D48, D66, D101 and D119) is enhanced, while the N-termini dissociation at lysine residue is greatly suppressed after dimethlylation for charge state of $14+$. For charge state of $16^{+}$, an enhancement of fragmentation at W28 to Y53 is observed after dimenthylation, while the fragmentation of the I66 to A90 is suppressed. However, the unlabeled and dimethylated lysozymes with a charge state of $18+$ exhibit little difference in the dissociation pattern. 

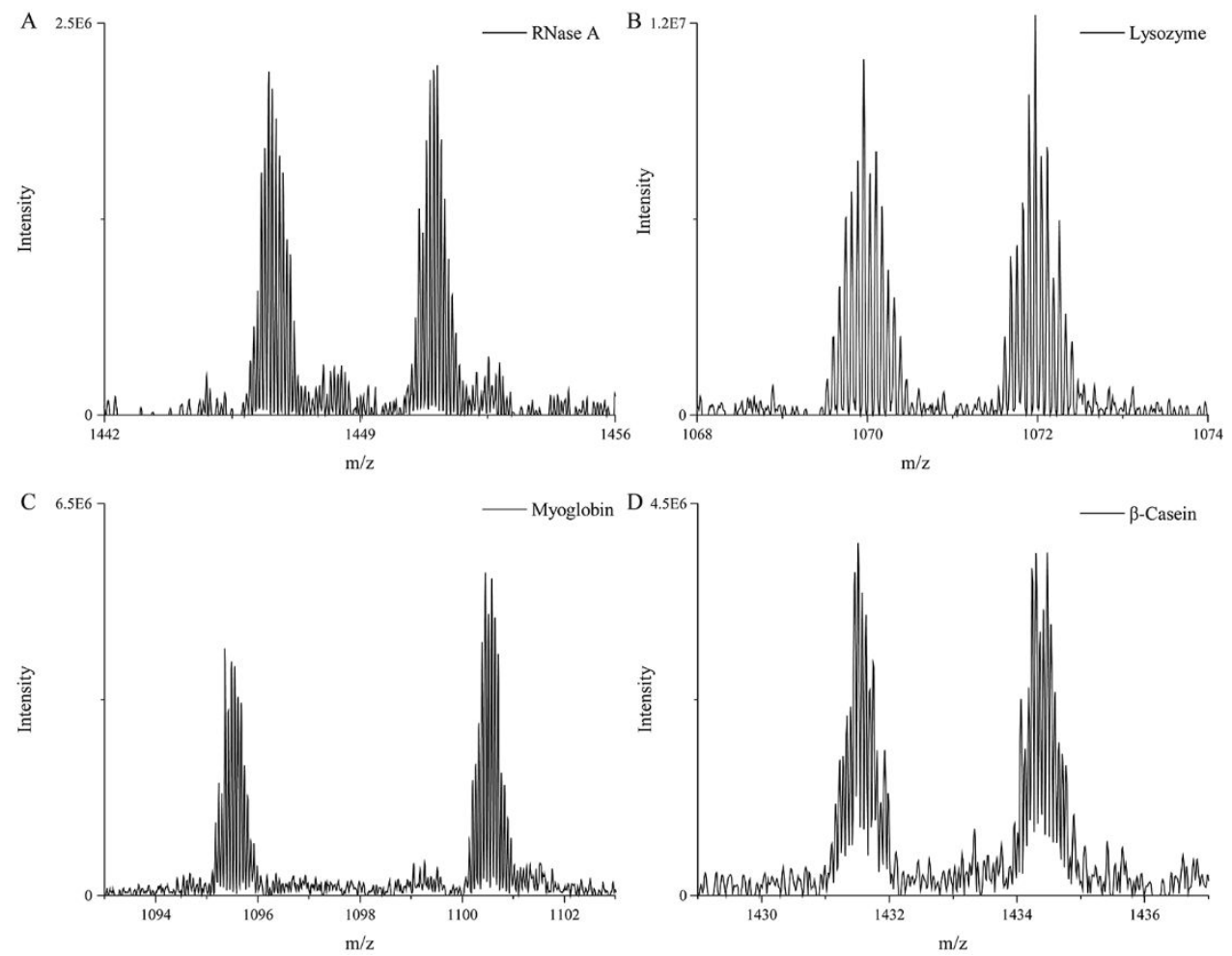

Figure S4 The MS spectra of dimethyl isotope labeled proteins (heavy/light=1), (A) RNase A; (B) lysozyme; (C) myoglobin and (D) $\beta$-casein. 
A

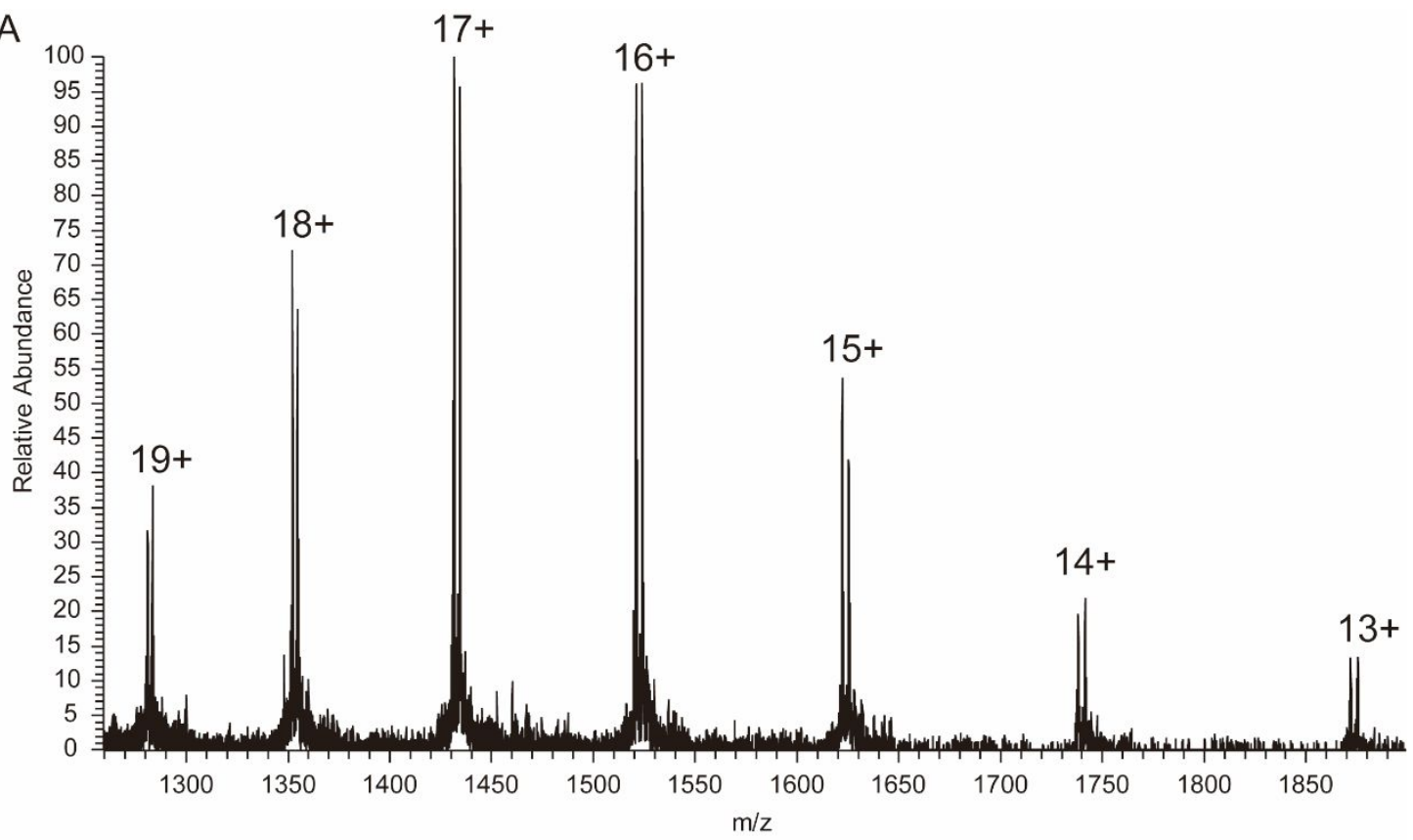

B

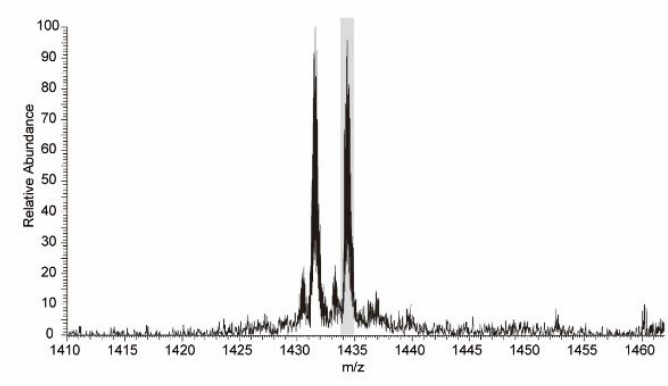

D

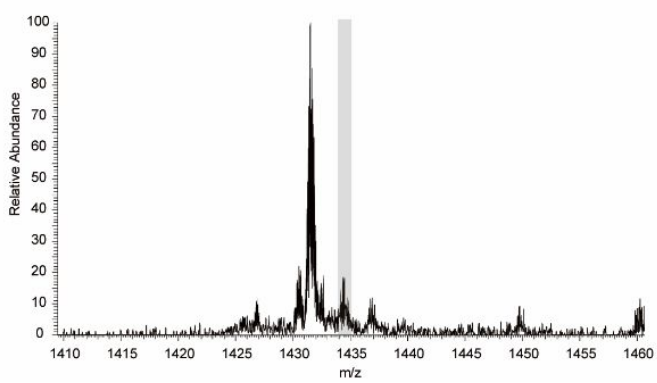

C

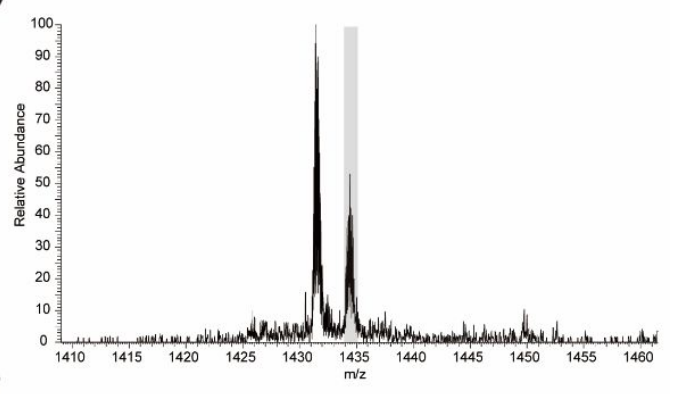

$\mathrm{E}$

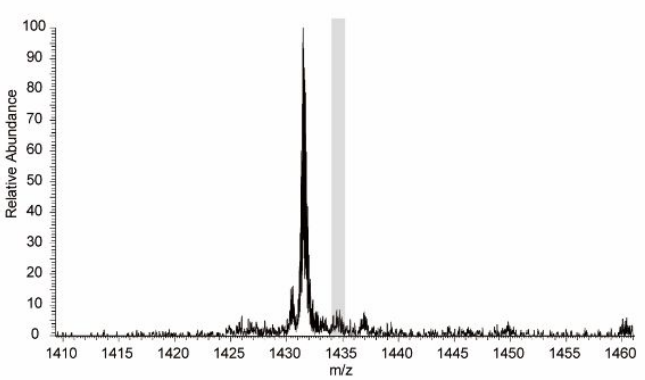

Figure S5 The MS spectra of dimethyl labeled $\beta$-casein. (A) the ion pairs with different charges in 1:1 pre-mixed sample; (B-E) the MS spectra of 1:1, 2:1, 5:1 and 10:1 with the charge state of 17+. 


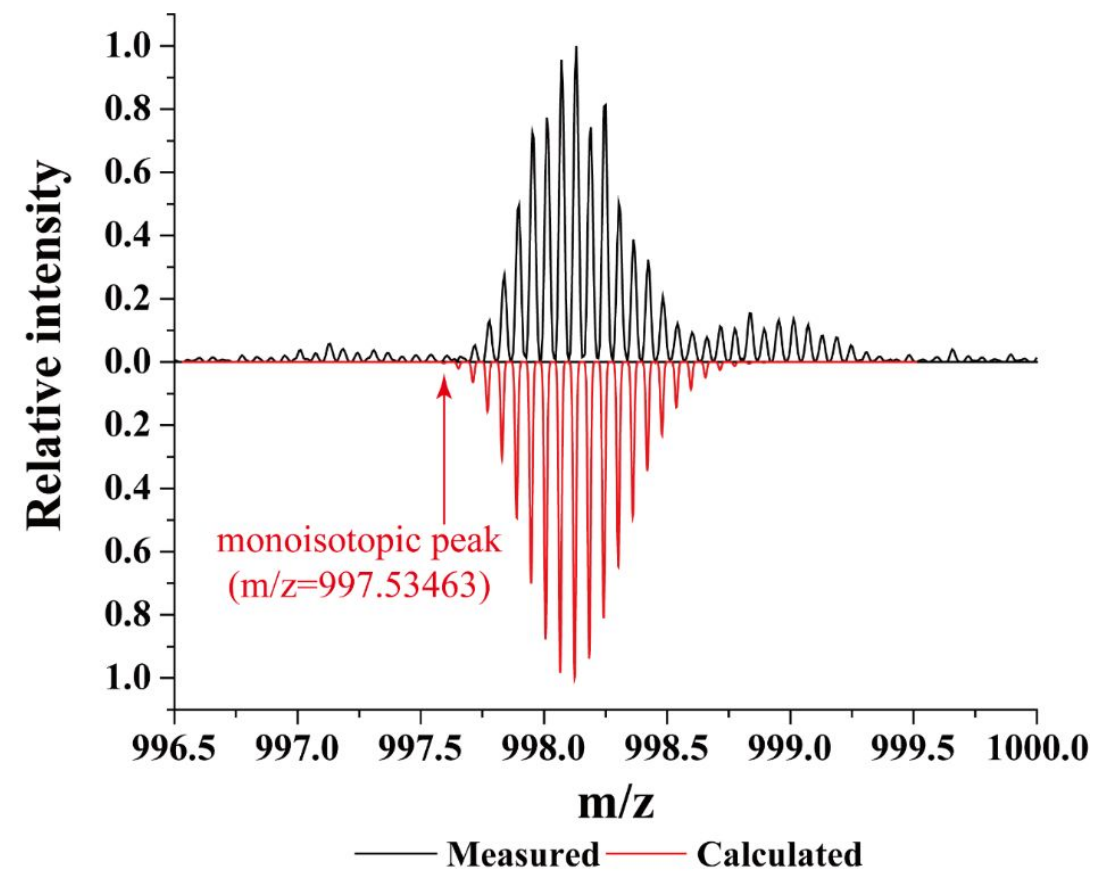

Figure S6 The measured and calculated MS spectra of myoglobin with a charge state of $17+$. The monoisotopic peak was indicated by red arrow.
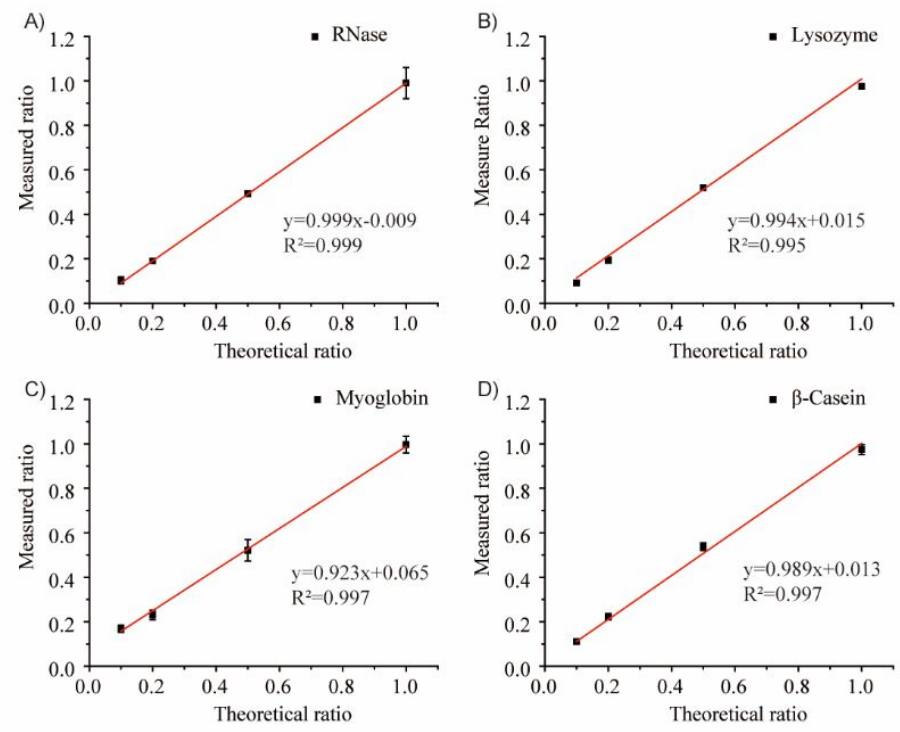

Figure S7 The linearity plot of quantitative ratio of each intact protein. 


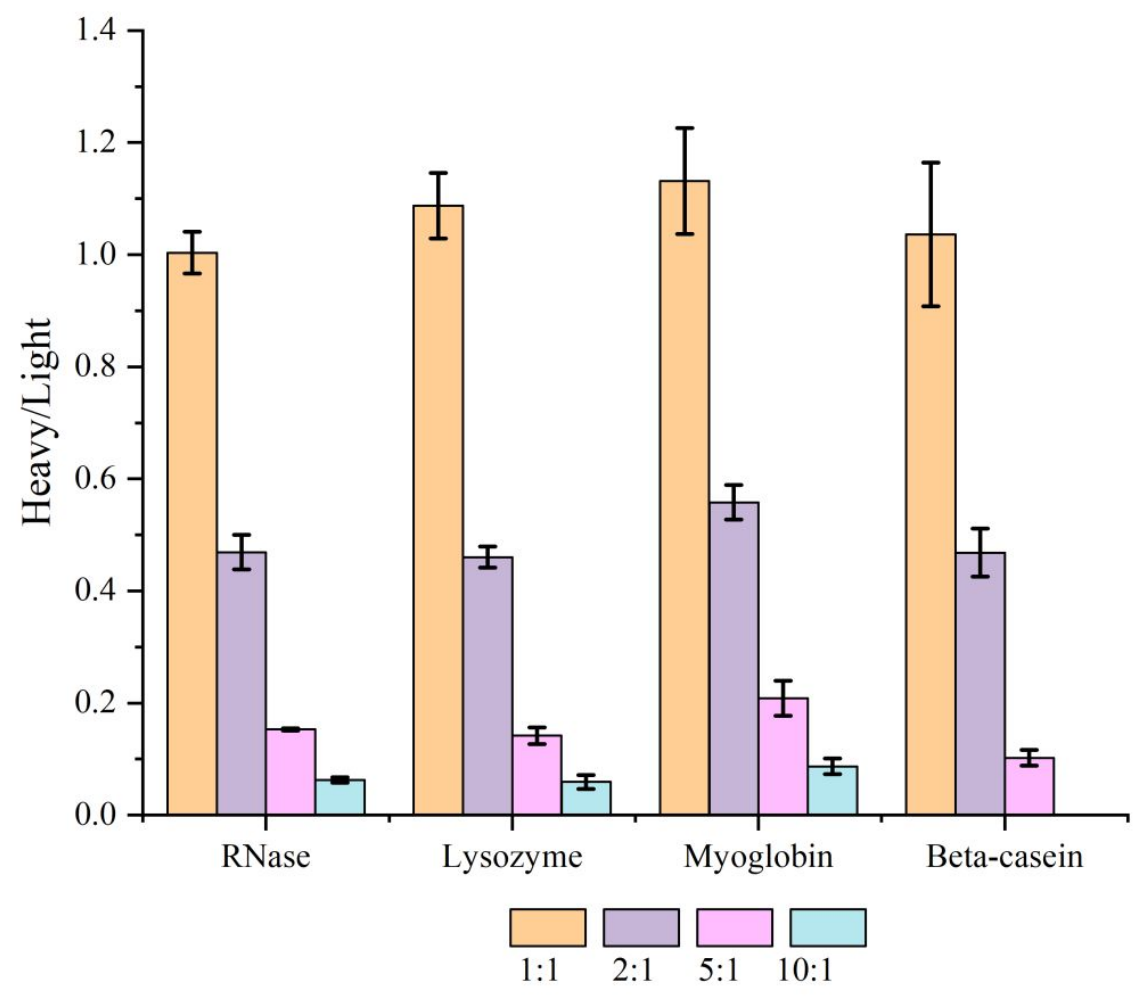

Figure $\mathrm{S} 8$ the quantification ratios of the mixed intact proteins with different pre-mixing ratios based on the intensities of monoisotopic species after deconvolution. In the monoisotopic specie-based quantification, the spectrum is firstly deconvoluted and transformed to a new spectrum consisted with all monoisotopic species, where the intensity of each monoisotopic peak is a sum of the intensity of all charge states belong to the same intact proteoform. Then, the intensities of light and heavy labeled proteoforms were utilized to get the quantification ratios. However, if the ratio is far away from 1, the numbers of the charge states of light and heavy labeled proteins are not equal, which would cause bias in quantification. As shown in the figure, for the protein sample with theoretical ratio of 1:10 (heavy/light), the measured ratio is $0.06,0.06,0.09$ and missed for RNase, lysozyme, myoglobin and $\beta$-casein, respectively. The corresponding ratios obtained with the intensity of the highest isotopic peak is $0.10,0.09,0.17$ and 0.11 (Figure 2B). Moreover, the light or heavy labeled protein may be missed during the step of deconvolution due to the low intensity. Exemplified by beta-casein in the standard protein mixture, the heavy labeled beta-casein is easily missed after deconvolution at a mix ratio of 1:10 (heavy/light), which caused the missing of quantification ratio. 

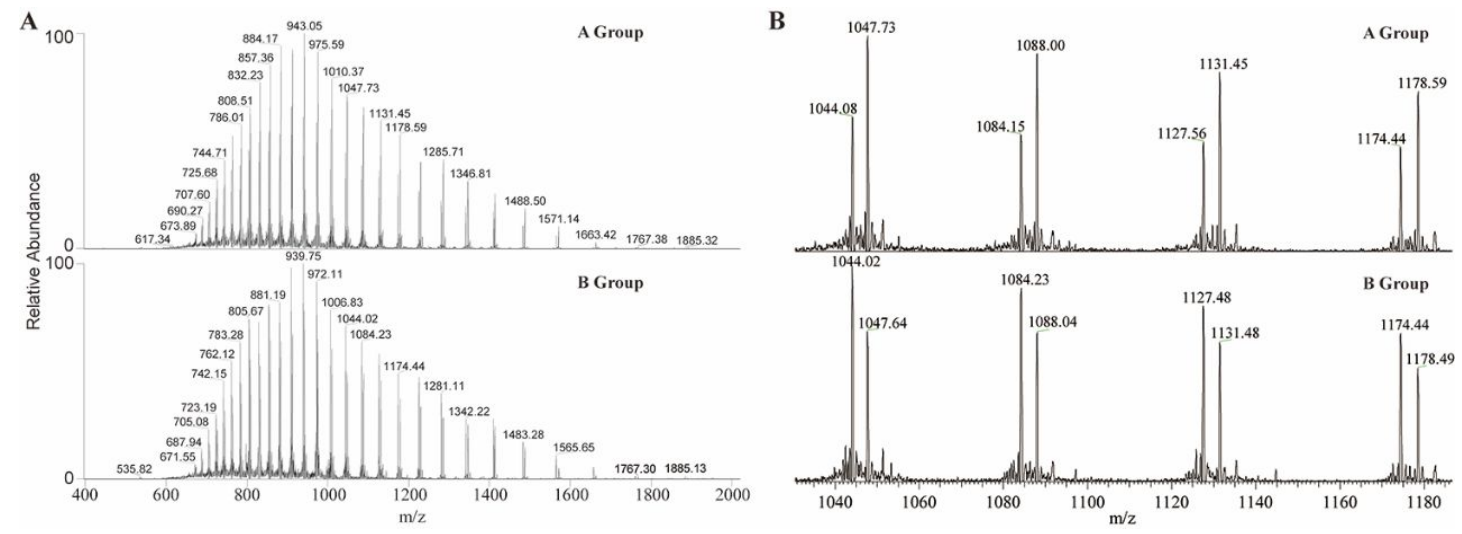

Figure S9 (A) MS1 spectra of an unknown protein with a mass of $28 \mathrm{kDa}$ detected in both A and B group; (B) the enlarge figures of MS1 spectra and a consistent result is obtained for this protein, which indicates that high quantification accuracy could well be obtained for large proteins.

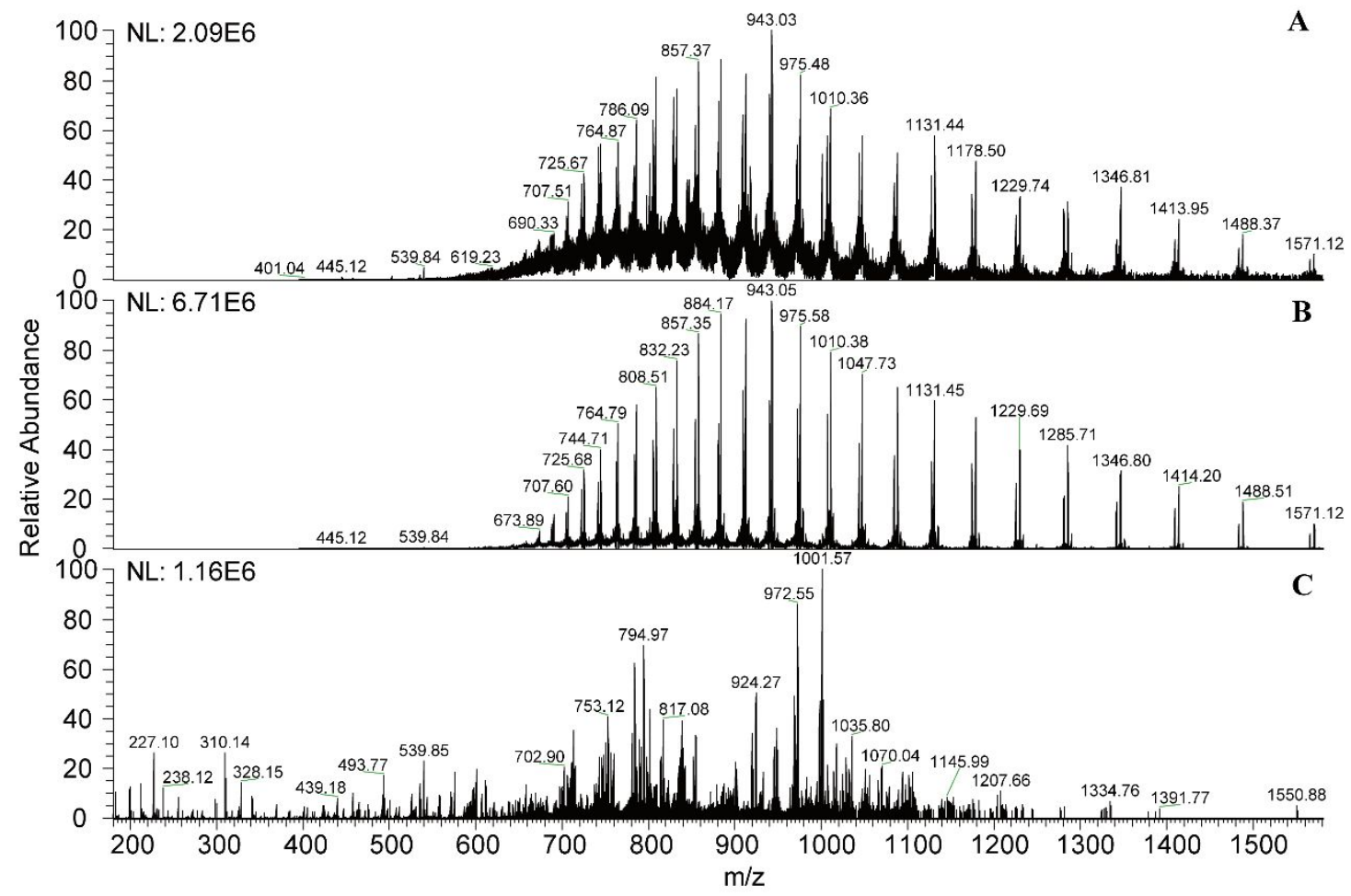

Figure S10 the MS1 and MS2 spectra of an unknown protein with a mass of $28 \mathrm{kDa}$. (A) MS1 spectrum obtained with a resolution of $70000(\mathrm{~m} / \mathrm{z}=200)$ and a micro-scan number of 3, (B) MS1 spectrum obtained with a resolution of $17500(\mathrm{~m} / \mathrm{z}=200)$ and a micro-scan number of 10 and $(C)$ MS2 spectrum of the protein ions with $\mathrm{m} / \mathrm{z}$ of 884.17. the spectra of MS1 were obtained by averaging 60 spectra in order to improve the $\mathrm{S} / \mathrm{N}$; a resolution of 70000 is not enough to distinguish the isotope envelope of this proteins, while a lower resolution could offer a higher signal intensity. 


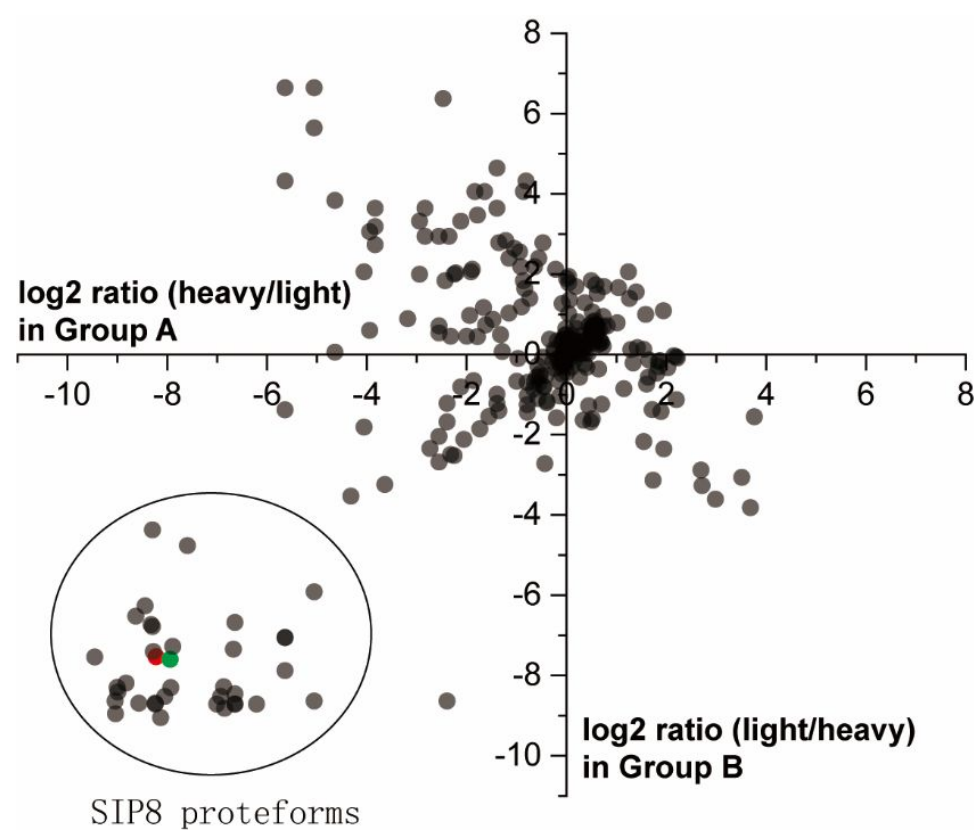

Figure S11 The correlation of quantification ratios ( $\log 2)$ for the proteoforms in groups A and B. Group A: anaerobic culture (light labeled) and aerobic culture (heavy labeled); Group B: anaerobic culture (heavy labeled) and aerobic culture (light labeled). Red dot and green dot indicate the SIP8 proteoforms without modification of oxidation. 

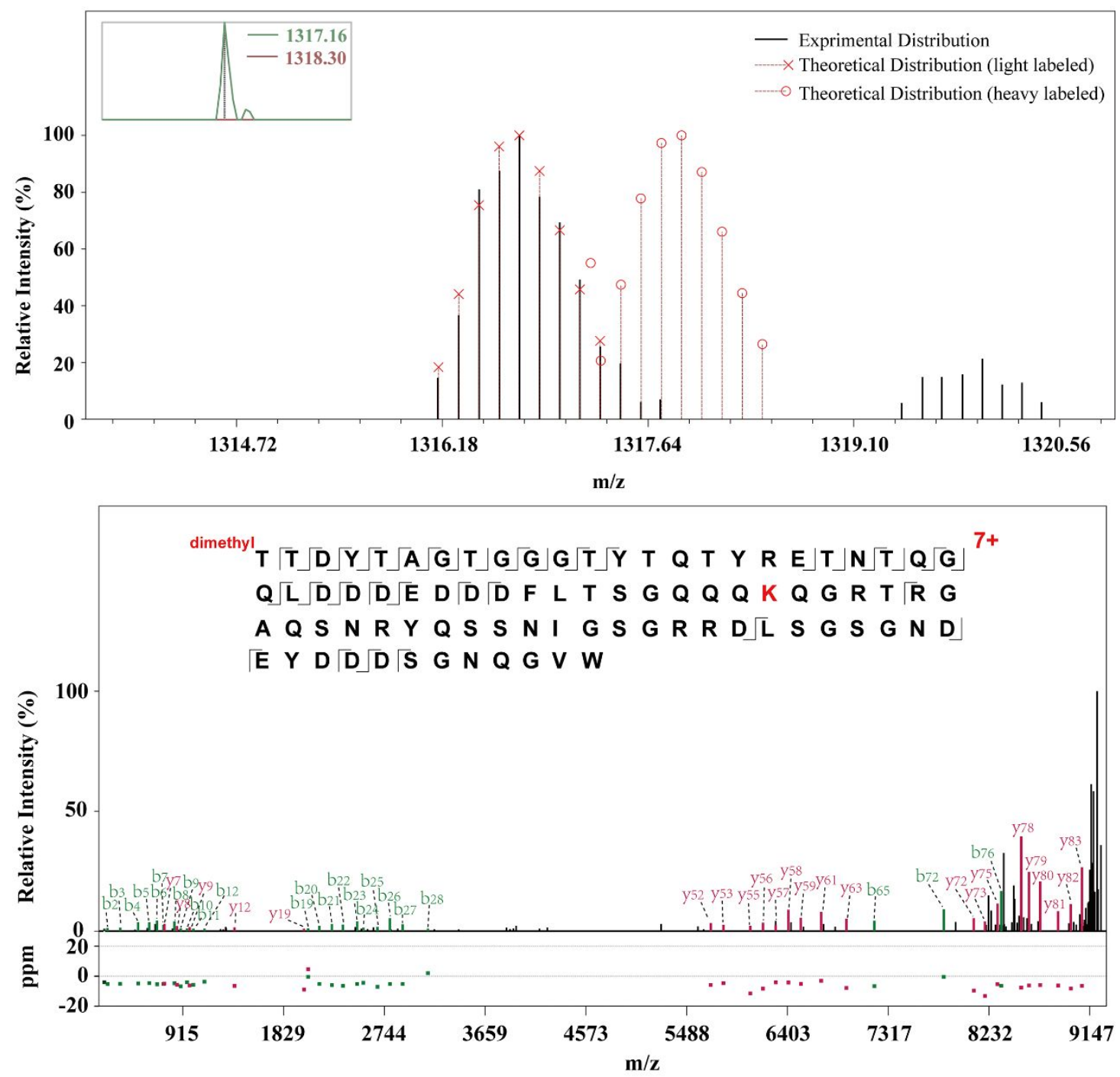

Figure S12 The quantification of GRE1 protein in Group A: (A), the MS1 spectra of GRE1 protein; (B), annotated and deconvoluted spectra of GRE1 protein; Group A: anaerobic culture (light labeled) and aerobic culture (heavy labeled). 

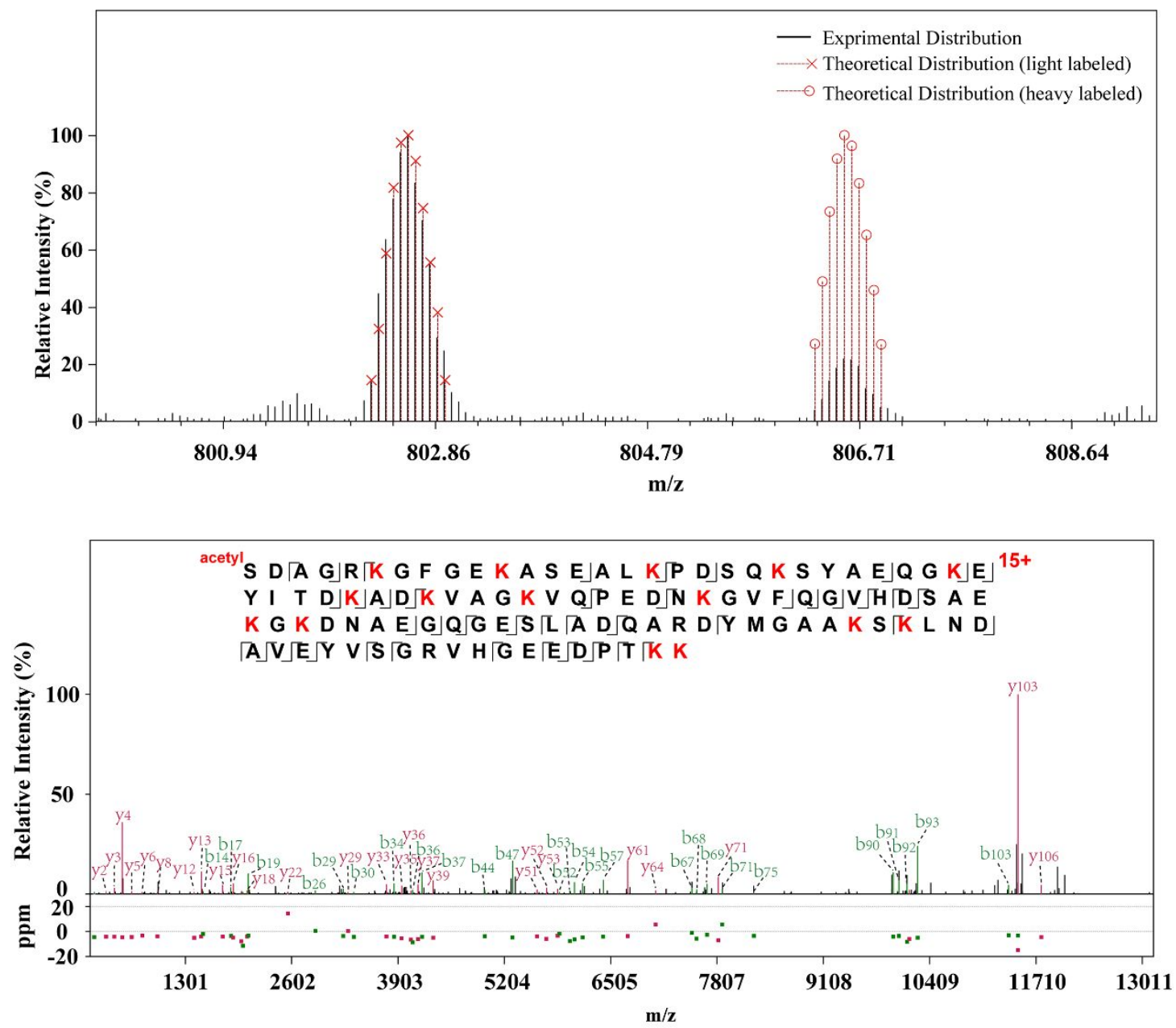

Figure S13 The quantification of HSP12 protein in Group A: (A), the MS1 spectra of HSP12 protein; (B), annotated and deconvoluted spectra of HSP12 protein; Group A: anaerobic culture (light labeled) and aerobic culture (heavy labeled). 
Table S2 The up-regulated and down-regulated proteins (anaerobic vs aerobic).

\begin{tabular}{|c|c|c|c|}
\hline \multicolumn{2}{|c|}{ Down-regulated } & \multicolumn{2}{|c|}{ Up-regulated } \\
\hline Protein Acession & Modifications & Protein Acession & Modifications \\
\hline sp|P38701|RS20_YEAST & |(0)Acetyl[ProteinN-term] & sp|P50263|SIP18_YEAST & |(0)Acetyl[ProteinN-term] \\
\hline sp|P38879|NACA_YEAST & & sp|Q08969|GRE1_YEAST & |(0)Acetyl[ProteinN-term] \\
\hline sp|Q3E7Y3|RS22B_YEAST & & sp|Q04438|SPG4_YEAST & \\
\hline sp|P33331|NTF2_YEAST & |(0)Acetyl[ProteinN-term] & sp|Q2V2P7|YD461_YEAST & $\mid(0)$ Acetyl[ProteinN-term $]$ \\
\hline sp|P07281|RS19B_YEAST & & sp|P01094|IPA3_YEAST & |(0)Acetyl[ProteinN-term] \\
\hline sp|P56628|RL22B_YEAST & & sp|P0CT04|IPB2_YEAST & \\
\hline sp|P50106|RPA14_YEAST & |(0)Acetyl[ProteinN-term] & sp|P22943|HSP12_YEAST & |(0)Acetyl[ProteinN-term] \\
\hline sp|Q3E754|RS21B_YEAST & |(0)Acetyl[ProteinN-term] & sp|Q3E841|YN034_YEAST & \\
\hline sp|P26786|RS7A_YEAST & |(0)Acetyl[ProteinN-term] & sp|P11633|NHP6B_YEAST & \\
\hline sp|P02400|RLA4_YEAST & & sp|P37299|QCR10_YEAST & \\
\hline \multirow[t]{3}{*}{ sp|P10622|RLA3_YEAST } & |(0)Acetyl[ProteinN-term] & sp|P0CX48|RS11B_YEAST & \\
\hline & & sp|P00445|SODC_YEAST & \\
\hline & & sp|P38804|SDO1L_YEAST & |(0)Acetyl[ProteinN-term] \\
\hline
\end{tabular}

